\title{
Prevalence and determinants of visual impairment amongst school-aged children in Southern Nigeria
}

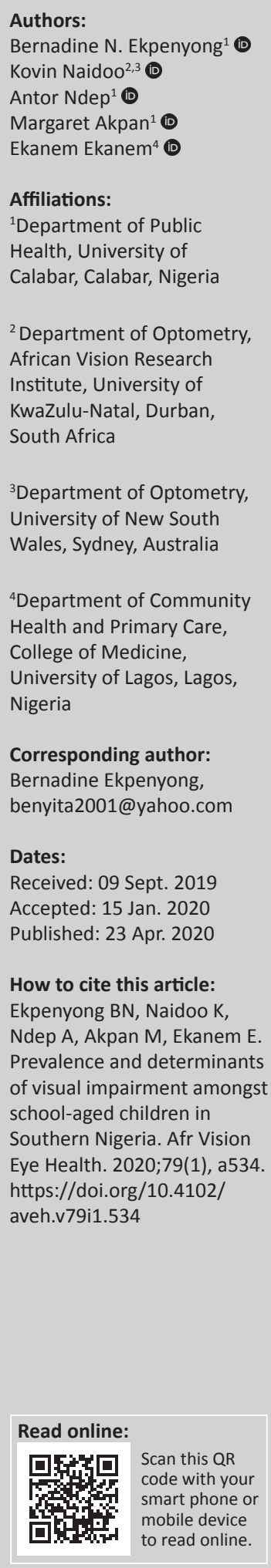

Background: Visual impairment among school children is relatively common and research provides evidence for integration of comprehensive eye care into school health programmes.

Aim: To determine the prevalence and determinants of visual impairment in school-aged children in Southern Nigeria.

Setting: School aged children from 10 public and private-owned schools in Cross River State, Southern Nigeria.

Methods: This cross-sectional analytic study used a multistage random sampling technique to select 2418 school children aged 6-17 years. Comprehensive eye examinations were performed on the study. The logistic regression analysis with $95 \%$ confidence interval (CI) was used to test hypotheses.

Results: Of the 2418 school children selected, 2110 (87\%) were assessed. The prevalence 'of uncorrected, presenting and best corrected visual acuity of $0.3(20 / 40)$ or worse in the better eye $^{\prime}$ was $7.3 \%, 7.2 \%$ and $0.19 \%$, respectively. Errors of refraction were the cause of the impairment in 198 (70.7\%; 63.5-76.0) eyes with reduced vision, followed by glaucoma suspects 38 (19.2\%; 13.8-24.8), amblyopia (3.0\%; 1.1-6.4) and corneal opacity $3(1.5 \% ; 0.3-4.3)$. The major and independent predictors of visual impairment were age (adjusted odds ratio [AOR] 1.97, 95\% 1.45-2.67), high socio-economic status (adjusted odds ratio [AOR] 1.68, 95\% 1.36-2.09) and female gender (AOR 1.35, 95\% 1.00-1.88).

Conclusion: The common causes of visual impairment in school-aged children are avoidable, and are mostly because of uncorrected refractive error, which could reflect inadequate refractive error services in the area.

Keywords: school-aged children; prevalence; refractive error; visual impairment.

\section{Introduction}

Vision problems, especially refractive error, amongst school-aged children are increasing globally. ${ }^{1,2}$ Low vision and blindness can impede growth in a child's education, particularly literacy and motor development impairment. ${ }^{3}$ The Global Action Plan 2010-2019 initiative has the target to reduce vision impairment (VI) by $25 \%$; therefore, there is a need to generate evidence to evaluate its success and other initiatives. ${ }^{4}$

Previously, we reported the prevalence of visual disorders of $32 \%$ in school children in Southern Nigeria and that only $1.8 \%$ of the children previously had an eye examination. ${ }^{5}$ As such, early diagnosis and treatment of physical and eye health conditions are not readily available because of implementation of the various policies developed by the Federal Ministry of Health in Nigeria.

There are numerous data available on visual impairment in school children in other countries. For example, the prevalence of uncorrected, presenting and best-corrected visual acuity (VA) of 20/40 or worse in the better eye, respectively, for school children in South Africa was $1.4 \%, 1.2 \%$ and $0.32 \%,{ }^{6}$ whereas for Ghana it was $3.7 \%, 3.5 \%$, and $0.4 \%$, respectively. ${ }^{7}$ In Southern India, the same findings were $2.7 \%, 2.6 \%$ and $2.6 \%{ }^{8}$; in China, it is $27 \%, 16.6 \%$ and $0.46 \%{ }^{9}$; and in Malaysia, it is $17.1 \%, 10.1 \%$ and $1.4 \% .{ }^{10}$ In all the studies reviewed, the highest cause of visual impairment was

Copyright: (C 2020. The Authors. Licensee: AOSIS. This work is licensed under the Creative Commons Attribution License. 
refractive error. Most of the studies in Nigerian schools reported only the overall prevalence of visual impairment in children and their definition of visual impairment varied widely. Studies on the prevalence of uncorrected, presenting and best corrected visual acuity of $20 / 40$ or worse in the better eye' for school children in Nigeria are unavailable to the best knowledge of the researcher, but are necessary information for effective school eye health programmes.

The main aim of this study was to determine the prevalence and determinants of visual impairment in school-aged children in Southern Nigeria. The results may be useful for planners of school health programmes in formulation of evidence-based policies and their implementation.

\section{Methods}

This cross-sectional analytic study design used a multistage random sampling technique in selecting 2418 children aged 6-17 years from 10 schools (both public and private) in Cross River State located in Southern Nigeria.

The eye examinations conducted on school children who participated in the study after informed consent from parents or caregivers included external and internal eye structure examinations, non-cycloplegic objective and subjective refraction. Visual acuity measurements at distance and near were done using a Logarithm of the minimum angle of resolution (LogMAR) chart. The sample size of children used for this study was computed using the formula ${ }^{11} n=\frac{Z^{2}(1-p)}{E^{2} p}$ and considering an estimated proportion of persons in the

population (school-age children) with eye problem as $15 \%{ }^{12}$ and $10 \%$ relative precision at a $95 \%$ confidence interval (CI). Allowance of $10 \%$ was made for non-responses and that gave a total sample size of 2418. Detailed data collection procedures as well as the sampling procedure used for this study can also be found in earlier publications. ${ }^{5,13}$

\section{Sampling procedure and methods of data collection}

The study was carried out in 10 selected schools from three local government areas (LGAs) in Cross River State. Seven public schools (4 primary, 3 secondary) and three private schools (2 primary, 1 secondary).

The first stage was the selection of one LGA from a list of LGAs in each of the three health zones in Cross River State using a simple random sampling technique by balloting, which yielded a total of three LGAs. The three LGAs selected were Calabar South in the south, Etung in the central and Ogoja in the north. The idea was to have a good spread that should be a true representation of the region concerned. In each of the selected LGAs, schools were stratified into public and private, primary and secondary schools. From the list of schools as already stratified, seven public schools (four primary and three secondary) and three private schools (two primary and one secondary) from the three selected LGAs were randomly selected for the study by balloting.

The next stage was the enrolment of children aged between 6 and 17 years from the participating schools. The population of each selected school was determined from the school register through the school head. Selection was done using simple random sampling based on a sampling probability proportionate to size. The total population of all the selected schools comprised 7922, which was used to determine the number of children enumerated for the study per school using simple proportions (school population / total population $\times$ sample size). Each school was made up of six class levels or grades with different arms per class level. One arm of each class level was randomly selected by balloting. A total of six class levels were selected per school and the total number to be enumerated per school as calculated was divided equally by six to represent each class level. The sampling frame used for the selection of children was a listing of class-based clusters within each school with the number of children in each. Only children who met the inclusion criteria (primary school children 6-11 years and secondary school children 12-17 years) were selected until the total required number was achieved per class level using the systematic sampling procedure. The next arm of the class level was generally selected if the first selected arm was not adequate.

Guidelines and recommendations from the Refractive Error Study in Children (RESC) were used in defining visual impairment ${ }^{14}$ as presenting VA of $\leq$ LogMAR 0.3 $(20 / 40)$ on the better eye and blindness as VA of $\leq$ LogMAR $1.0(20 / 200)$. However, the study modified some other recommendations of the RESC, like the use of noncycloplegic refraction procedure, which also informed the definition of hyperopia used for this study. The fogging technique was used in place of cycloplegic refraction to achieve a similar relaxation of accommodation in the school children. ${ }^{15}$ Significant refractive error was defined as myopia of $\geq|-0.50 \mathrm{D}|$, astigmatism of $\geq|-0.75 \mathrm{D}|$ and hyperopia of $\geq 1.50 \mathrm{D}$.

Assent was obtained verbally from the participants after a detailed briefing of the procedure before the procedures started, in addition to a written consent from the parents or guardians. Data were collected using intervieweradministered semi-structured questionnaires, after which participants' eyes were examined to determine the eye health status using a battery of procedures, which included VA assessment using LogMAR VA charts for distance and near vision; external eye examination with the pentorch; internal eye examination with the direct ophthalmoscope; noncycloplegic objective refraction using an autorefractor and retinoscope; subjective refraction with $2 \mathrm{D}$ fogging lens; cover test via the cover-uncover technique, at distance and at near, respectively; and colour vision test using an Ishihara test chart to determine the presence or absence of colour abnormality. Diagnosis was determined based on the anatomical and aetiological considerations. Cup to disc ratio 
of 0.5 and above was used to diagnose glaucoma suspects. Refractive error was given as the cause of reduced vision for all eyes correcting to LogMAR $0.2(20 / 32)$ or better with subjective refraction, even when other contributing diseases were present.

Independent replicate examinations for quality assurance were carried out in four schools using $5 \%$ of the sample size to validate the data collected. Details of quality assurance measures are presented in an earlier publication. ${ }^{5}$

\section{Data analysis}

The analysis of data was performed using Statistical Package for Social Sciences (SPSS) version 20. Socioeconomic status (SES) of each child's family was constructed from a combination of parents' occupation and education. Parents with no formal education were scored as 0 , and those with primary, secondary and tertiary education were scored as 1, 2 and 3, respectively. Occupational categories were also scored accordingly. From the composite scores, parents were classified into low, medium and high SES. For the purpose of logistic regression analysis, dichotomous categorical variables were recoded to 0 and 1 , where 0 referred to the reference category and 1 referred to the other category. Socioeconomic status was treated as an ordinal variable. The hypothesis on the relationship between independent variables (age, sex, school type and SES) and the occurrence of visual impairment in school-aged children while controlling for potential confounders was examined using unconditional logistic regression analysis.

The parents or caregivers of the study participants provided consent through a consent form sent home with their children or wards. Assent was also obtained from the school children before participation in the study.

\section{Ethical considerations}

Ethical clearance to conduct the study was obtained from the Cross River State Health Research Ethics Committee (CRS-HREC) (clearance number CRS/MH/CGS/E-H/018/ VOLII/081).

\section{Results}

A sample size of 2110 (87.3\% response rate) children aged 6-17 years, from both public (government-owned) and private schools was evaluated. More than half of the children $(52.9 \%)$ were females, $59.2 \%$ were in primary schools (6-11-year-olds) and 76.4\% were in public schools (Table 1). Visual acuity is an important outcome measure for assessing vision. This measurement was possible in 2105 of 2110 children examined, leaving five children not assessed because of lack of cooperation. Visual acuity measurements were obtained in $99.7 \%$ of males and $99.8 \%$ of females. Uncorrected VA of LogMAR $0.2(20 / 32)$ or better in one or both eyes was found in 1951 (92.7\%) children. Of these children, 928 (93.7\%)
TABLE 1: Socio-demographic characteristics of school-aged children.

\begin{tabular}{lcc}
\hline Variables & Frequency $(\boldsymbol{n}=\mathbf{2 1 1 0})$ & $\%$ \\
\hline Sex & 993 & 47.1 \\
Male & 1117 & 52.9 \\
Female & & \\
Age (years) & 1250 & 59.2 \\
6-11 (primary) & 860 & 40.8 \\
12-17 (secondary) & & \\
LGA & 942 & 44.6 \\
Calabar south & 419 & 19.9 \\
Etung & 749 & 35.5 \\
Ogoja & & \\
School type & 1612 & 76.4 \\
Public & 498 & 23.6 \\
Private &
\end{tabular}

LGA, local government area.

were males and $1023(91.7 \%)$ were females (Table 2). Those with uncorrected vision of LogMAR $0.3(20 / 40)$ or worse in the better eye were 154 (7.3\%) children. Four $(0.2 \%)$ had VA of LogMAR $0.1(20 / 200)$ or worse in the better eye and three of them were females (Table 2).

Three of the children presented with spectacles during the vision assessment, and only one case showed improvement in VA with spectacles. The 'prevalence of presenting visual acuity in the better eye of LogMAR $0.3(20 / 40)$ or worse' was $153(7.2 \%)$ and the prevalence of VA LogMAR 1.0 or worse did not change; it was still four as in the uncorrected VA. Children with VA of LogMAR $0.2(20 / 32)$ or better after best optical correction increased from 1939 (92.1\%) to 2101 (99.8\%). No child was found in the LogMAR 1.0 (20/200) or worse VA category. Overall, the prevalence of visual acuity of 0.3 $(20 / 40)$ or worse' decreased to $4(0.19 \%)$ with optical correction (Table 3).

The major causes of uncorrected vision LogMAR 0.3 (20/40) or worse in one or both eyes in the 198 eyes included refractive error 140 (70.7\%; CI 63.5-76.0), glaucoma suspects 38 (19.2\%; CI 13.8-24.8), amblyopia 6 (3.0\%; CI 1.1-6.4), corneal opacity $3(1.5 \%$; $0.3-4.3)$, phthisis bulbi $3(1.5 \%$; CI $0.3-4.3)$, cataract and retinal anomaly $1(0.5 \%$; CI $0.0-2.8)$ each, and others that included cases of developmental abnormalities such as micro-ophthalmia, ptosis, strabismus and other neurological conditions, unknown cases and injury (6,3\%; CI 1.1\%-6.4\%) (Table 4).

Parents' or guardians' highest level of education and occupation were used as a proxy to measure their SES into high, medium and low SES. From the logistics regression analysis (Table 5), age group, sex, SES and school type were found to be significantly associated with visual impairment. Children aged 12-17 years were two times more likely to have presenting VA of LogMAR 0.3 or worse than those aged 6-11 years. Females were more likely than males to have a visual impairment. Children whose parents are within the medium and high SES were about two times more likely to have impaired vision than those whose parents were in the low socio-economic class. This means that the risk of visual impairment was significantly associated with SES of parents. 
TABLE 2: Distribution of uncorrected, presenting and best-corrected visual acuity by gender.

\begin{tabular}{|c|c|c|c|c|c|c|c|c|c|c|c|c|}
\hline \multirow[t]{3}{*}{ VA category } & \multicolumn{4}{|c|}{ Uncorrected VA } & \multicolumn{4}{|c|}{ Presenting VA } & \multicolumn{4}{|c|}{ Corrected VA } \\
\hline & \multicolumn{2}{|c|}{ Boys } & \multicolumn{2}{|c|}{ Girls } & \multicolumn{2}{|c|}{ Boys } & \multicolumn{2}{|c|}{ Girls } & \multicolumn{2}{|c|}{ Boys } & \multicolumn{2}{|c|}{ Girls } \\
\hline & $n$ & $\%$ & $n$ & $\%$ & $n$ & $\%$ & $n$ & $\%$ & $n$ & $\%$ & $n$ & $\%$ \\
\hline$\geq 0.2(20 / 32)$ both eyes & 925 & 93.4 & 1014 & 90.9 & 925 & 93.4 & 1014 & 90.9 & 982 & 99.2 & 1103 & 99.0 \\
\hline$\geq 0.2(20 / 32)$ either of the two eyes & 3 & 0.3 & 9 & 0.8 & 3 & 0.3 & 10 & 0.9 & 6 & 0.6 & 10 & 0.9 \\
\hline$\leq 0.30(20 / 40)$ to $\geq 0.5(20 / 63)$ better eye & 55 & 5.6 & 85 & 7.6 & 55 & 5.6 & 84 & 7.5 & 1 & 0.1 & 2 & 0.2 \\
\hline$\leq 0.60(20 / 80)$ to $\geq 0.9(20 / 160)$ better eye & 6 & 0.6 & 4 & 0.4 & 6 & 0.6 & 4 & 0.4 & 1 & 0.1 & 0 & 0.0 \\
\hline$\leq 1.0(20 / 200 ; 6 / 60)$ better eye & 1 & 0.1 & 3 & 0.3 & 1 & 0.1 & 3 & 0.3 & 0 & 0.0 & 0 & 0.0 \\
\hline Total & 990 & - & 1115 & - & 990 & - & 1115 & - & 990 & - & 1115 & - \\
\hline
\end{tabular}

Visual impairment: $\leq 0.30$ LogMAR (20/40) or worse in the better eye; Blind: $\leq 1.0$ LogMAR (20/200; 6/60) or worse in the better eye.

VA, visual acuity.

TABLE 3: Uncorrected, presenting and best-corrected visual acuity of school-aged children.

\begin{tabular}{|c|c|c|c|c|c|c|c|c|c|}
\hline \multirow[t]{2}{*}{ VA category } & \multicolumn{3}{|c|}{ Uncorrected VA } & \multicolumn{3}{|c|}{ Presenting VA } & \multicolumn{3}{|c|}{ Corrected VA } \\
\hline & $n$ & $\%$ & $95 \% \mathrm{Cl}$ & $n$ & $\%$ & $95 \% \mathrm{Cl}$ & $n$ & $\%$ & $95 \% \mathrm{Cl}$ \\
\hline$\geq 0.2(20 / 32)$ both eyes & 1939 & 92.1 & $90.9-93.2$ & 1939 & 92.1 & $90.9-93.2$ & 2085 & 99.0 & $98.5-99.4$ \\
\hline$\geq 0.2(20 / 32)$ either of the two eyes & 12 & 0.6 & $0.3-1.0$ & 13 & 0.6 & $0.3-1.0$ & 16 & 0.8 & $0.5-1.3$ \\
\hline$\leq 0.30(20 / 40)$ to $\geq 0.5(20 / 63)$ better eye & 140 & 6.7 & $5.6-7.8$ & 139 & 6.6 & $5.6-7.8$ & 3 & 0.1 & $0.0-0.5$ \\
\hline$\leq 0.60(20 / 80)$ to $\geq 0.9(20 / 160)$ better eye & 10 & 0.5 & $0.2-0.9$ & 10 & 0.5 & $0.2-0.9$ & 1 & 0.05 & $0.0-0.3$ \\
\hline$\leq 1.0(20 / 200 ; 6 / 60)$ better eye & 4 & 0.2 & $0.1-0.5$ & 4 & 0.2 & $0.1-0.5$ & 0 & 0.0 & - \\
\hline Total & 2105 & - & - & 2105 & - & - & 2105 & - & - \\
\hline
\end{tabular}

Visual impairment: $\leq 0.30$ LogMAR (20/40) or worse in the better eye; Blind: $\leq 1.0$ LogMAR $(20 / 200 ; 6 / 60)$ or worse in the better eye.

$\mathrm{VA}$, visual acuity; $\mathrm{Cl}$, confidence interval.

TABLE 4: Causes of uncorrected visual acuity 0.30 LogMAR (20/40) or worse.

\begin{tabular}{|c|c|c|c|c|c|c|c|c|}
\hline \multirow[t]{2}{*}{ Causes } & \multicolumn{2}{|c|}{ Right eyes $n=186$} & \multicolumn{2}{|c|}{ Left eyes $n=181$} & \multicolumn{2}{|c|}{ One or both eyes $n=198$} & \multirow{2}{*}{$\begin{array}{l}\text { Prevalence } \\
95 \% \mathrm{Cl}\end{array}$} & \multirow{2}{*}{$\begin{array}{c}\text { Prevalence } \\
\text { Total }\end{array}$} \\
\hline & $n$ & $\%$ & $n$ & $\%$ & $n$ & $\%$ & & \\
\hline Refractive error & 135 & 72.6 & 131 & 72.4 & 140 & 70.7 & $63.5-76.0$ & 6.6 \\
\hline Glaucoma & 34 & 18.3 & 37 & 20.4 & 38 & 19.2 & $13.8-24$. & 1.8 \\
\hline Amblyopia & 6 & 3.2 & 5 & 2.8 & 6 & 3.0 & $1.1-6.4$ & 0.28 \\
\hline Corneal opacity & 1 & 0.5 & 1 & 0.6 & 3 & 1.5 & $0.3-4.3$ & 0.14 \\
\hline Cataract & 1 & 0.5 & 0 & 0.0 & 1 & 0.5 & $0.0-2.8$ & 0.05 \\
\hline Retinal anomaly & 1 & 0.5 & 0 & 0.0 & 1 & 0.5 & $0.0-2.8$ & 0.05 \\
\hline Phthisis bulbi & 2 & 1.1 & 2 & 1.0 & 3 & 1.5 & $0.3-4.3$ & 0.14 \\
\hline Other causes & 6 & 3.2 & 5 & 2.8 & 6 & 3.0 & $1.1-6.4$ & 0.28 \\
\hline
\end{tabular}

Note: The total cause for the cause-specific prevalence (198) exceeds the any cause prevalence (154). This is so because children with VA LogMAR 0.3 (20/40) or worse in both eyes may represent two different causes of reduced vision. Other causes include developmental abnormalities like micro-ophthalmia, ptosis, strabismus, other neurological conditions, unknown conditions and injury.

$\mathrm{Cl}$, confidence interval.

TABLE 5: Visual impairment determinants in school-aged children.

\begin{tabular}{lccccccc}
\hline Variable & OR & $\mathbf{9 5 \%}$ & $\mathrm{Cl}$ & Coefficient & SE & Z-Statistic & $\boldsymbol{p}$-value \\
\hline Age group & 2.3220 & 1.1505 & 4.6862 & 0.8424 & 0.3583 & 2.3513 & 0.0187 \\
Sex & 1.4196 & 1.0354 & 1.9462 & 0.3503 & 0.1610 & 2.1762 & 0.0295 \\
SES (2/1) & 2.2279 & 1.5032 & 3.3022 & 0.8011 & 0.2008 & 3.9900 & 0.0001 \\
SES (3/1) & 2.4362 & 1.5090 & 3.9333 & 0.8905 & 0.2444 & 3.6434 & 0.0003 \\
Sch_type & 1.8630 & 1.2992 & 2.6714 & 0.6222 & 0.1839 & 3.3833 & 0.0007 \\
\hline
\end{tabular}

SES, socio-economic status; OR, odds ratio; Sch type, school type (public and private schools); $\mathrm{Cl}$, confidence interval; $\mathrm{SE}$, standard error.

Children who attended private schools were more likely to have visual impairment than those in the public schools $(p<0.001$, Table 5).

\section{Discussion}

Vision impairment in this study was defined according to recommendations of the RESC as presenting VA of $\leq$ LogMAR $0.3(20 / 40)$ in the better eye and blindness as VA of $\leq \log$ MAR $1.0(20 / 200)$. This was done so as to compare the results of this research with other standard RESC. The prevalence 'of uncorrected, presenting and best-corrected VA of LogMAR $0.3(20 / 40)$ or worse on the better eye' was $7.3 \%$, $7.2 \%$ and $0.19 \%$, respectively. The results of studies in other countries were lower than the results of this study; South Africa had 1.4\%, 1,2\% and $0.32 \%$; Ghana had 3.7\%, 3.5\% and $0.4 \%{ }^{16}$; and India had 2.7\%, 2.6\% and 2.6\% ${ }^{17}$ Best-corrected vision amongst the school-aged children was $0.19 \%$, which implies that about $98 \%$ of the causes of visual impairment are correctable with spectacles and this suggests unavailability of eye care services in the country.

In this study, all the children enrolled were refracted irrespective of VA, which could account for the higher prevalence of visual impairment reported, when compared with other studies in Nigeria, Ghana and South Africa. ${ }^{6,718,19}$

However, higher percentages for countries such as China $(27 \%, 16.6 \% \text { and } 0.46 \%)^{9}$ and Malaysia $(17.1 \%, 10.1 \% \text { and } 1.4 \%)^{10}$ were reported in the literature. The difference in results is not unconnected with the variation in study areas, definitions of visual impairment and research protocols. What is rather consistent in all the studies is that about two-thirds of the visual impairment in school-aged children were because of refractive error, followed by amblyopia. $6,7,18,19,20$ 
The main causes of VI found in this study were refractive error $(70.7 \%)$, which is a treatable cause of VI; glaucoma suspects $(38 \%)$ and amblyopia (3.0\%), which can usually be prevented through early diagnosis and treatment; corneal opacity $(1.5 \%)$, which is also a preventable and treatable cause of VI; cataract $(0.5 \%)$, which is treatable through surgery; and retinal anomaly $(0.5 \%)$ that may be preventable via early diagnosis. Similar studies in Nigeria also found errors of refraction to be the main cause of impaired vision in school-aged children. ${ }^{12,21,22,23,24}$

Two cases of monocular phthisis bulbi (also preventable) were seen in a particular LGA located in the central senatorial district of Cross River State, which requires further investigation to establish its relationship with the environmental condition of the area. The number of children who fell into the vision criteria of 1.0 LogMAR or worse was $4(0.2 \%)$, and three them were girls. No child met the category of 'presenting visual acuity of less than 1.3 LogMAR in the better eye'. Monocular blindness which is defined as vision criteria of 1.0 LogMAR or worse in one eye was 20 $(0.95 \%)$. In this study, the prevalence of blindness is similar to results from other studies, ${ }^{6,12,18}$ although the definition of blindness used in these studies varied considerably.

The low prevalence of blindness found in this study and others could be attributed to the fact that most school children with blindness may likely stay at home to avoid stigmatisation. ${ }^{6}$ This therefore calls for a population-based visual impairment study in children. After best correction, the overall prevalence of presenting VA of $0.3 \operatorname{LogMAR}$ $(20 / 40)$ or worse decreased from $153(7.2 \%)$ to $4(0.19 \%)$, which may imply that the delivery of accessible refractive error services with provision of affordable spectacles would significantly reduce the magnitude of VI in schoolaged children.

Children of school age whose parents were classified as high SES were more likely than those with low SES to have VI. This is closely linked with the association between school type (private vs. public) and prevalence of refractive error; those in private schools were two times more likely to have VI $(p<0.001)$ than those in the public schools. Private schools almost always have higher school or tuition fees when compared to public schools; therefore, parents in higher SES tend to be more likely to afford to send their children to private schools. A similar study in Calabar Municipality comparing students from private and public secondary schools also found that refractive errors were significantly more amongst children whose parents are in the high socio-economic class. ${ }^{25}$ Studies carried out elsewhere also found that children of parents of upper to middle SES, as well as children in private and urban schools, have higher refractive error. ${ }^{26,27}$ However, other studies found no significant relationship between these variables. ${ }^{28,29}$ Significantly, the prevalence of VI was more amongst the girls than the boys $(p<0.01)$. This could be because of the fact that about $70 \%$ of those with VI also had refractive error. Girls have been known to be more involved in activities that require near work than boys, like reading and doing house chores, while boys are more likely to engage in outdoor activities. ${ }^{5}$ Older children (12-17 years old) were more likely to have vision of 0.3 LogMAR or worse than children aged 6-11 years. Similar studies found the same result. ${ }^{26,30,31}$ The reason may be that older children engage in more near work, and also refractive error (myopia) increases with age. In the absence of adequate resources, eye care programme targeted at school children could focus on older children 12 years and above.

\section{Conclusion}

The major causes of visual impairment amongst schoolaged children were refractive error, glaucoma suspects, amblyopia and corneal opacity. Most of these causes are preventable and about $99.8 \%$ of them are correctible with spectacles, and only three out of those who need spectacles wore them; this implies that the uptake of refractive services amongst school-aged children is low. Without screening programmes at schools, children with visual problems may go undetected. Provision of spectacles will greatly reduce the prevalence of impaired vision amongst school-aged children. Age, SES of parents, sex and school type were the independent predictors of VI in school children. Government policy on eye and physical health examination and treatment before admittance to school and periodically during school years is recommended and school eye health should be fully integrated into school health programmes.

\section{Acknowledgements}

The authors would like to thank Prof. Hannah Faal for contributing ideas during the proposal stage of the research, and the University of Calabar and the University of Calabar Teaching Hospital for providing enabling environment and some equipment for the study.

\section{Competing interests}

The authors have declared that no competing interests exist.

\section{Authors' contributions}

B.N.E. was the lead researcher and was responsible for the conceptualisation of the research. K.N. supervised the study and gave the research direction. A.N. and M.A. participated in data collection. E.E. provided professional supervision and guidance and also assisted in the data analysis.

\section{Funding information}

Funding for this research was provided by the African Vision Research Institute, South Africa.

\section{Data availability statement}

The data for this study is original and own creation and available to team members. 


\section{Disclaimer}

The views and opinions expressed in this article are those of the authors and do not necessarily reflect the official policy or position of any affiliated agency of the authors.

\section{References}

1. Naidoo KS, Leasher J, Bourne RR, et al. Global vision impairment and blindness due to uncorrected refractive error, 1990-2010. Optom Vis Sci. 2016;93(3): 227-234. https://doi.org/10.1097/OPX.0000000000000796

2. Pascolini D, Mariotti SP. Global estimates of visual impairment: 2010. Br J Ophthalmol 2012;96(5):614-618. https://doi.org/10.1136/bjophthalmol-2012-301861

3. Holden BA, Fricke TR, Wilson DA, et al. Global prevalence of myopia and high myopia and temporal trends from 2000 through 2050. Ophthalmology. 2016;123(5):1036-1042. https://doi.org/10.1016/j.ophtha.2016.01.006

4. WHO. Towards universal eye health: A global action plan 2014-2019. Resolution of the Sixty-sixth World Health Assembly [homepage on the Internet]. 2013 [cited 2018 Apr 02]. Available from: https://apps.who.int/iris/bitstream/ handle/A66 R4-en

5. Ekpenyong BN, Naidoo K, Ahaiwe $\mathrm{K}$, et al. Visual status and prevalence of eye disorders among school-age children in southern Nigeria. Afr Vis Eye Health. 2017;76(1):1-6. https://doi.org/10.4012/aveh.v7611.377

6. Naidoo KS, Raghunandan A, Mashige KP, et al. Refractive error and visual impairment in African children in South Africa. Invest Ophthalmol Vis Sci. 2003;44(9):3764-3770. https://doi.org/10.1167/iovs.03-0283

7. Kumah BD, Ebri A, Abdul-Kabir M, et al. Refractive error and visual impairment in private school children in Ghana. Optom Vis Sci. 2013;90(12):1456-1461. https:// doi.org/10.1097/OPX.000000000000099

8. Dandona $\mathrm{R}$, Dandona $\mathrm{L}$, Srinivas $\mathrm{M}$, et al. Refractive error in children in a rura population in India. PubMed Commons. Investig Ophthalmol Vis. 2002;43(3): 615-637.

9. He $M$, Huang $W$, Zheng $Y$, Huang L, Ellwein L. Refractive error and visual impairment in school children in rural southern China. Ophthalmology [serial online]. 2007 [cited 2015 Aug 6]. Available from: http://www.sciencedirect.com/science/ [cited 2015 Aug 6]. Available
article/pii/S0161642006011389

10. Goh P, Abqariyah Y, Pokharel G, Ellwein L. Refractive error and visual impairment in school-age children in Gombak District, Malaysia. Ophthalmology [serial in school-age children in Gombak District, Malaysia. Ophthalmology [serial
online]. 2005 [cited 2015 Aug 6]. Available from: http://www.sciencedirect.com/ science/article/pii/S0161642004017956

11. Lwanga SK, Lemeshow S. Sample size determination in health studies: A practical manual. World Heal Organ Bull. 1991;9(11):1-80.

12. Ajaiyeoba Al, Isawumi MA, Adeoye AO, Oluleye TS. Prevalence and causes of blindness and visual impairment among school children in south-western Nigeria. Int Ophthalmol [serial online]. 2007;26(4-5):121-125. https://doi.org/10.1007/ s10792-005-4836-4

13. Ekpenyong BN, Naidoo K, Ndep AO, et al. Comparative analysis of satisfaction with the use of ready-made spectacles and custom-made spectacles among schoo children in Nigeria: A randomised controlled trial. J Heal J. 2017;35:15-21.

14. Resnikoff S. Assessment of the prevalence of visual impairment attributable to refractive error or other causes in school children. Protocol and manual of procedures. Geneva: World Health Organization; 2016.
15. Queiros A, Gonzalez-Meijome JJ. Influence of fogging lenses and cycloplegia on open field automatic refraction. Ophthalmic Physiol Opt. 2008;29(4):387-392. https://doi.org/10.1111/j.1475-1313.2008.00579.x

16. Kumah D, Owusu E, Kyeremaa F. Prevalence of hyperopia among school children in the Kumasi metropolis, Ghana. J Ghana Sci Assoc. 2012;14(1):63-68.

17. Dandona R, Dandona L. Childhood blindness in India: A population based perspective. Br J Ophthalmol. 2003;87(3):263-265. https://doi.org/10.1136/bjo. 87.3.263

18. Okoye O, Umeh RE, Ezepue FU. Prevalence of eye diseases among schoo children in a rural south-eastern Nigerian community. Rural Remote Health. 2013;13(3):2357.

19. Nartey ET, Van Staden DB, Amedo AO. Prevalence of ocular anomalies among schoolchildren in Ashaiman, Ghana. Optom Vis Sci. 2016 Jun 1;93(6):607-611 https://doi.org/10.1097/OPX.0000000000000836

20. Alrasheed SH, Naidoo KS, Clarke-Farr PC. Prevalence of visual impairment and refractive error in school-aged children in South Darfur State of Sudan. Afr Vision Eye Health. 2016;75(1):1-13. https://doi.org/10.4102/aveh.v75i1.355

21. Opubiri I, Pedro-Egbe C. Screening for refractive error among primary school children in Bayelsa state, Nigeria. Pan Afr Med J. 2013; 22:129-134. https://doi. org/10.11604/pamj.2013.14.74.1345

22. Ayanniyi AA, Mahmoud AO, Olatunji FO. Causes and prevalence of ocula morbidity among primary school children in Ilorin, Nigeria. Niger J Clin Pract. 2010;13(3):248-253

23. Abah ER, Oladigbolu KK, Samaila E, Gani-lkilama A. Ocular disorders in children in Zaria children's school. Niger J Clin Pract. 2011;14(4):473-476. https://doi. org/10.4103/1119-3077.91759

24. Kehinde AV, Ogwurike SC, Eruchalu UV, Pam V, Samaila E. School eye health screening in Kaduna - Northern Nigeria. Niger J Surg Res. 2005;7(1):191-194. https://doi.org/10.4314/njsr.v7i1.12276

25. Osuchukwu NC, Osuchukwu EC, Akpabio I, Ekpenyong B. Socio-economic background and prevalence of visual defects amongst students in public and private seconary schools in Calabar Municipality. Glob J Pure Appl Sci. 2013; 19(2):151-155.

26. Sapkota YD, Adhikari BN, Pokharel GP, Poudyal BK, Ellwein LB. The prevalence of visual impairment in school children of upper-middle socioeconomic status in Kathmandu. Ophthalmic Epidemiol. 2008;15(1):17-23. https://doi.org/10.1080/ 09286580701772011

27. Padhye AS, Khandekar R, Dharmadhikari S, Dole K, Gogate P, Deshpande M. Prevalence of uncorrected refractive error and other eye problems among urban and rural school children. Middle East Afr J Ophthalmol. 2009;16(2):69-74. https://doi.org/10.4103/0974-9233.53864

28. Uzma N, Kumar BS, Salar BMKM, Zafar MA, Reddy VD. A comparative clinical survey of the prevalence of refractive errors and eye diseases in urban and rural school children. Can J Ophthalmol. 2009;44(3):328-333. https://doi.org/10.3129/ i09-030

29. Shrestha RK, Joshi MR, Ghising R, Rizyal A. Ocular morbidity among children attending government and private schools of Kathmandu valley. J Nepal Med Assoc. 2011;51(184):182-188. https://doi.org/10.31729/jnma.21

30. Hashim SE, Tan HK, Wan-Hazabbah WH, Ibrahim M. Prevalence of refractive error in Malay primary school children in suburban area of Kota Bharu, Kelantan, Malaysia. Ann Acad Med Singapore. 2008;37(11):940-946.

31. Paudel $P$, Ramson $P$, Naduvilath $T$, et al. Prevalence of vision impairment and refractive error in school children in Ba Ria - Vung Tau province, Vietnam. Clin Exp Ophthalmol. 2014;42(3):217-226. https://doi.org/10.1111/ceo.12273 